\title{
Quantifying the projected unit cell size variation of off-axis PtCo catalyst nanoparticles through 4D-STEM
}

\author{
Debangshu Mukherjee ${ }^{1}$, Haoran $\mathrm{Yu}^{2}$, Chenyu Wang $^{3}$, Jacob Spendelow ${ }^{4}$, David Cullen ${ }^{5}$ and Michael \\ Zachman $^{5}$
}

${ }^{1}$ Center for Nanophase Materials Sciences, Oak Ridge National Laboratory, KNOXVILLE, Tennessee, United States, ${ }^{2}$ Center for Nanophase Materials Sciences, Oak Ridge National Laboratory, Oak Ridge, Tennessee, United States, ${ }^{3}$ Materials Physics and Applications Division, Los Alamos National Laboratory, New Mexico, United States, ${ }^{4}$ Materials Physics and Applications Division, Los Alamos National Laboratory, Los Alamos, New Mexico, United States, ${ }^{5}$ Center for Nanophase Materials Sciences, Oak Ridge National Laboratory, Tennessee, United States

The role of surface strain in modifying catalytic activity is an active area of investigation since it is is a measure of the inter-atomic distances. Changing the inter-atomic lengths modifies the orbital overlap, shifting the d-band center and modifying the surface-adsorbate reactions' strength [1, 2,3]. An increase in the d-band center to a higher energy results in stronger interactions, while lowering the energy does the opposite. Of the multitude of techniques available to the materials scientist, four-dimensional scanning transmission electron microscopy (4D-STEM) uniquely offers both sub-picometer strain precision and resolutions below unit cell lengths $(\sim 0.5 \mathrm{~nm})$, as compared to conventional methods such as geometric phase analysis (GPA), and atomic-resolution STEM [4, 5, 6, 7]. Since strain in 4D-STEM is conventionally measured by tracking the evolution of the diffraction disks' location, which correspond to known crystallographic axes, one significant challenge in this technique is that the data must be taken with the sample oriented on a known low-index zone axis. This issue afflicts conventional EM strain measurement techniques, too, since atom columns need to be visible in order to locate them. Thus, the sample needs to be tilted to the relevant zone axis before data acquisition. For single-crystal samples, this is often straightforward. However, for real-world samples such as metal nanoparticles randomly oriented on a carbon support structure, as in fuel cell and electrolyzer catalysts, this can be a time-consuming task that limits the throughput of the strain quantification.

All 4D-STEM strain metrology techniques, where the data is collected only from a single specimen tilt [8, 9], calculate a projected strain from an averaged diffraction pattern as the beam travels through the sample being investigated. Here, we demonstrate a technique for estimating the projected unit cell size variation of a material that does not lie on a low-index zone axis, enabling measurements to be performed across multiple particles, with the entire workflow implemented in the STEMTool Python package [10]. Fig. 1(a) shows a single PtCo nanoparticle's bright-field image on a carbon support film tilted $\sim 15$ degrees from a zone-axis orientation. Fig. 1(b) shows the mean diffraction pattern for this particle and reveals that a small number of diffraction disks are still present, even when oriented off-axis. Strain mapping can, therefore, still be performed, provided that the diffraction disks present, including the central disk, are noncollinear. The diffraction patterns are processed by applying a Sobel filter and cross-correlating the resulting pattern with the edge of the central diffraction disk, based on a technique previously demonstrated [6]. The processing results in an array of Gaussian peaks, as shown in Fig. 1(c), the location of which is identified with sub-pixel precision by a peak finding algorithm and then fitting them with two-dimensional gaussian distributions. This procedure is first performed on the mean diffraction pattern, followed by the data at each scan position on the particle. Comparing the diffraction disk locations between the individual probe position and mean data allows the projected unit cells' size expansion or contraction to be calculated compared to the mean pattern. Since the particle is not required to be oriented on-axis, strain directions $\left(\varepsilon_{\mathrm{xx}}, \varepsilon_{\mathrm{xy}}, \varepsilon_{\theta}\right.$ and $\left.\varepsilon_{\mathrm{yy}}\right)$ are chosen arbitrarily, with the directions in Fourier space shown in the schematics in Fig. 1(d). This results in strain maps as in Fig. 1(e)-Fig. 1(h), with directions in real space corresponding to the schematic shown in Fig. 1(i). While these individual strain maps are challenging to interpret due to the arbitrarily chosen strain directions, the observed crystal expansion is more easily interpretable and can be calculated based on the following equation, where $\varepsilon_{\mathrm{vol}}$ is the change in projected unit cell size: 
$\varepsilon_{\mathrm{vol}}=\left(\left(1+\varepsilon_{\mathrm{xx}}\right) \times\left(1+\varepsilon_{\mathrm{yy}}\right) \times \cos \left(\varepsilon_{\theta}\right)\right)-1$

Fig. 1(j) shows the corresponding change in the projected unit cell size $\left(\varepsilon_{\mathrm{vol}}\right)$, as calculated from the individual strain directions based on the above equation and reveals a strained outer shell compared to the particle core.

Thus, this technique allows the projected unit cell size variation to be calculated from off-axis particles, which we exploit to measure multiple nanoparticles with different orientations in a single data set. Fig. 2(a) shows five PtCo particles on a carbon support film with unknown orientations. Using particle finding approaches, we first identify regions of the data corresponding to each of the particles. Using the method described above, we then successfully measure the projected unit cell size variation of three of the particles, as demonstrated in Fig. 2(b), despite these particles having various off-axis orientations, as can be seen in their mean diffraction patterns in Fig. 2(c)-Fig. 2(e). In each particle, we find a strained outer shell compared to the particle core, similar to the particle shown in Fig. 1(j). This work, therefore, demonstrates how 4D-STEM strain mapping can be extended to off-axis particles, which enables measurements to be obtained from particles in multiple orientations within a single dataset. Thus, this capability will help perform statistically relevant numbers of strain measurements, allowing a better understanding of how the unit cell size varies across many particles oriented in different directions. This technique thus removes the rate-limiting step for strain mapping 4D-STEM, something that is still present in conventional EM techniques, and allows high-precision unit cell comparisons across systems with multiply oriented grains such as battery electrodes, sputtered thin films, etc.
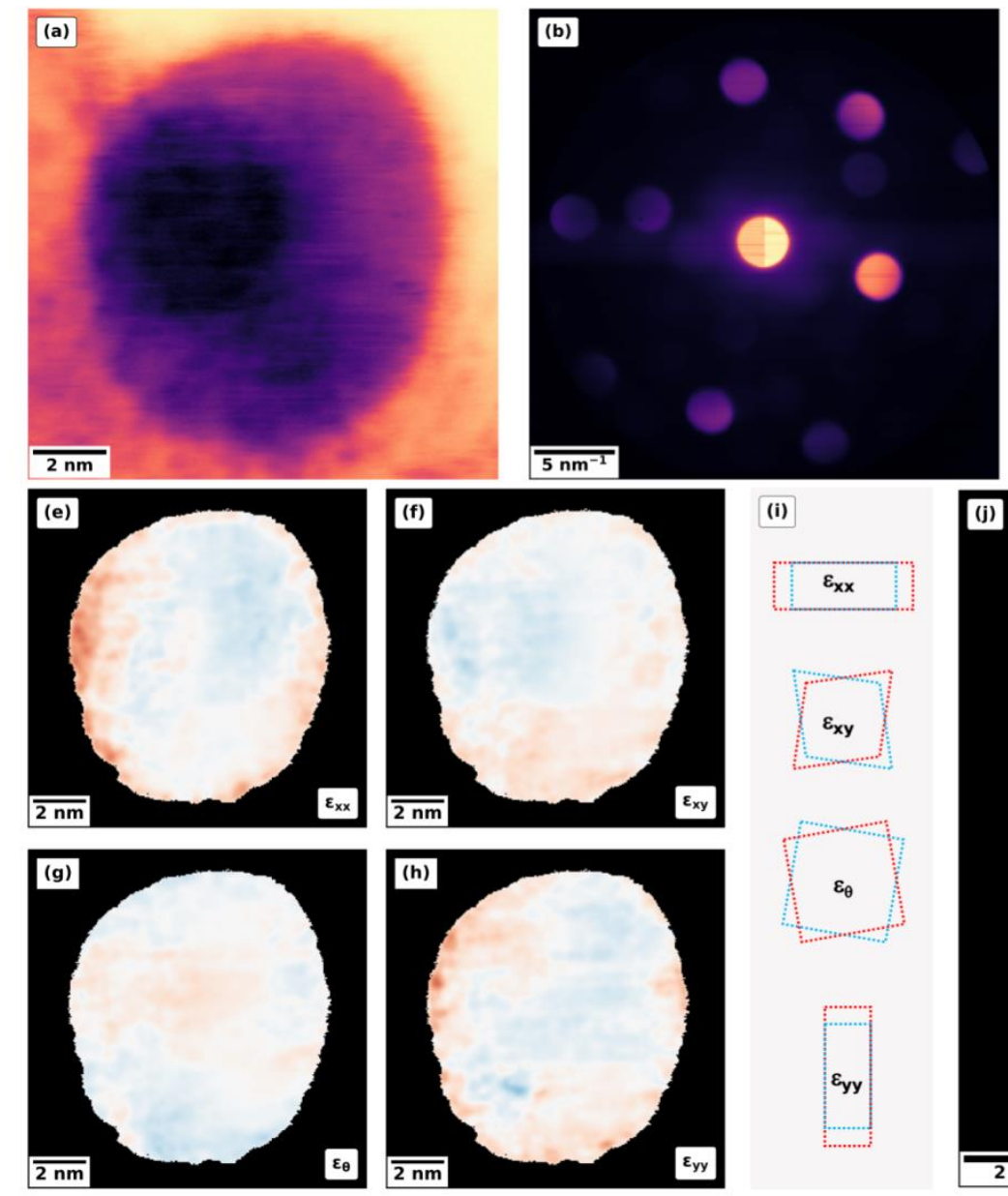
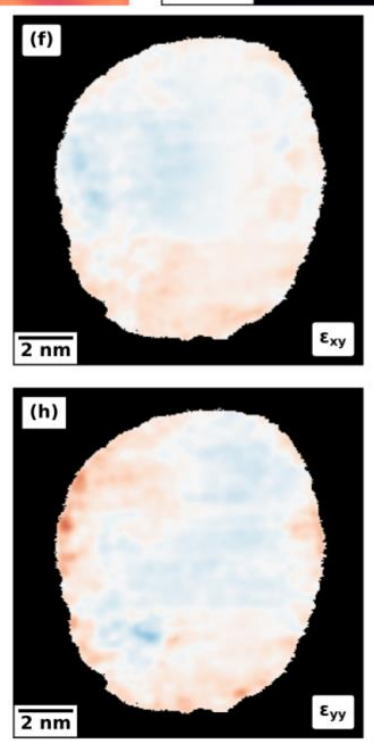
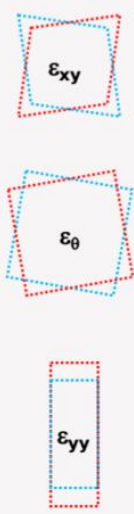
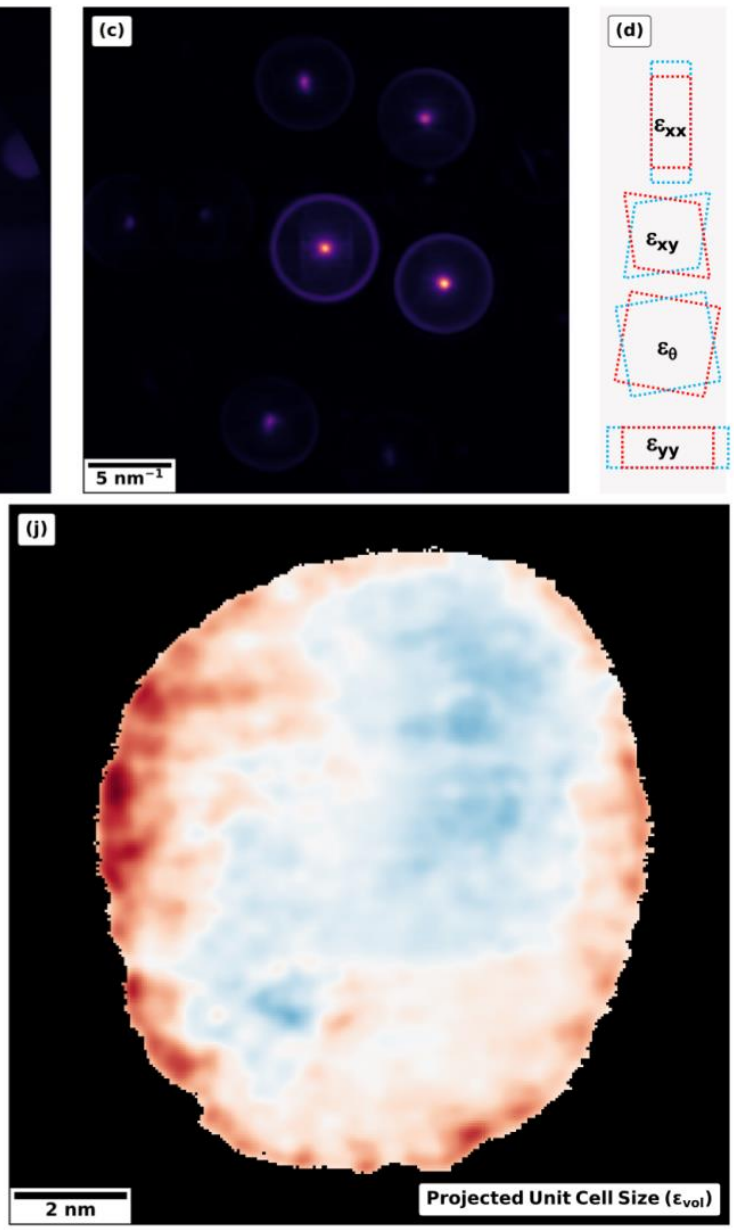

Strain (\%)

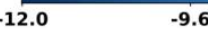

$-7.2$

$-4.8$

2.4

4.8

7.2

9.6 12.0 
Figure 1. Measuring strain from an off-axis PtCo nanoparticle. (a) Synthetic BF-STEM image of a PtCo fuel cell catalyst nanoparticle on a carbon support, obtained by summing the diffraction patterns at every scan position. This particle is tilted approximately 150 from the zone axis. (b) Mean diffraction pattern from the particle. (c) Cross-correlation between the edge of a diffraction disk with the pre-filtered CBED pattern demonstrates how the diffraction disks are transformed to peaks whose positions can be estimated to determine the disk locations with sub-pixel precision. (d) The arbitrarily chosen strain directions $(\Sigma \mathrm{xx}, \Sigma \mathrm{xy}, \Sigma($ and $\Sigma \mathrm{yy}$ respectively) when measuring in the diffraction (Fourier) space. (e) - (h) Individual strain values based on the arbitrarily chosen directions. (i) Representation of the arbitrarily chosen strain directions in real space. Compared to the Fourier directions, $\Sigma x x$ and $\Sigma y y$ are reversed in orientation. Also, the directions of the strain magnitudes are reversed, as a compression in Fourier space is expansion in real space, and vice versa. (j) Projected unit cell size variation $\left(\sum \varpi 0 \lambda\right)$ of the particle calculated from the individual strain values. Blue regions indicate contraction with respect to the mean, while red areas indicate expansion. As can be observed, the lattice at the particle's surface is expanded with respect to its interior.

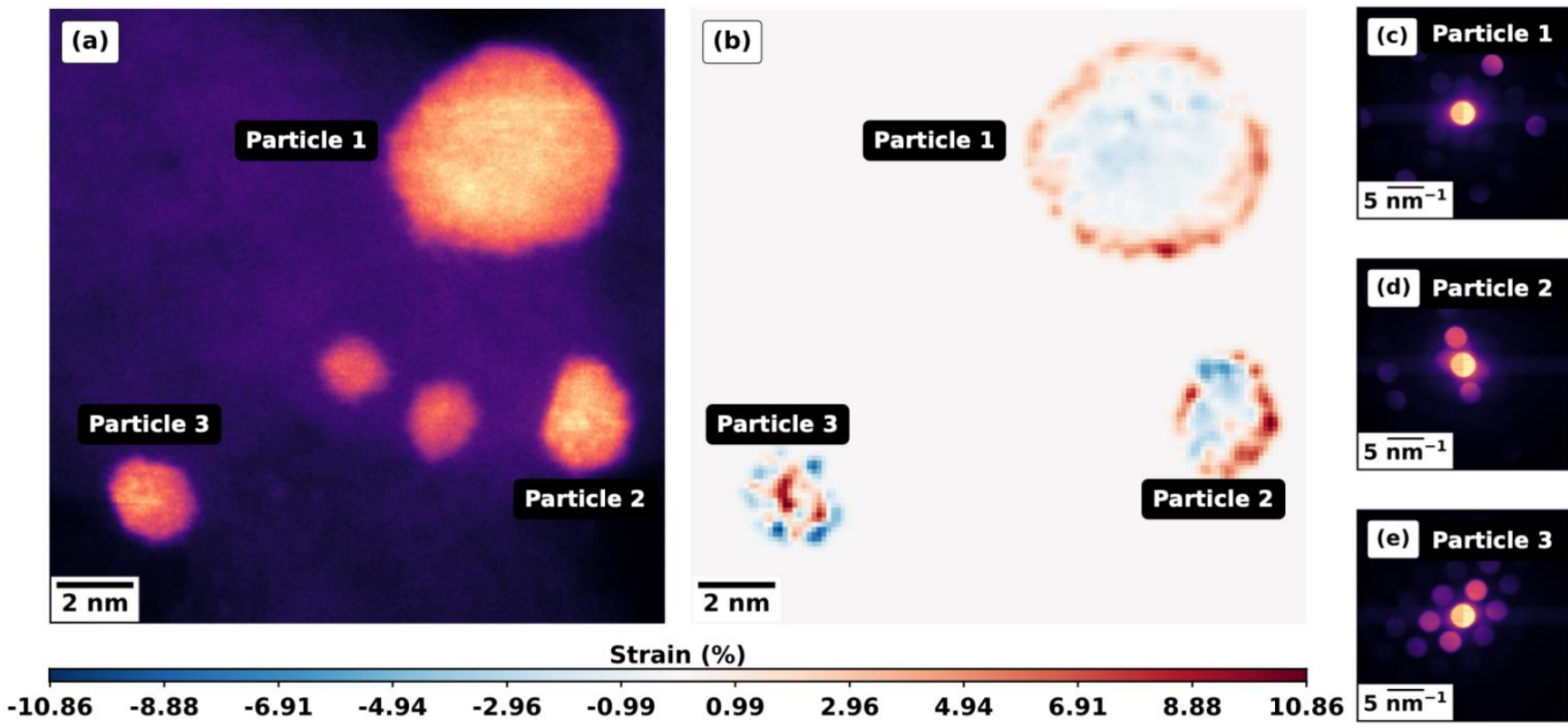

Figure 2. Measuring strain from multiple particles simultaneously. (a) Simultaneous ADF-STEM image of a cluster of PtCo nanoparticles on a carbon support. (b) Projected unit cell size variation for three of the particles (marked as Particles 1, 2, and 3) calculated from the 4D-STEM diffraction patterns. (c) - (e) Mean diffraction patterns from the measured particles, showing the particles' various orientations. As in Fig. 1(j), the particles' surfaces are strained compared to their cores.

References

[1] S Zhang et al., J. Am. Chem. Soc. 136 (2014), p. 7734.

[2] M Mavrikakis et al., Phys. Rev. Lett. 81 (1998), p. 2819.

[3] P Moseley et al., Nano Lett. 15 (2015), p. 4089.

[4] K Müller et al., Appl. Phys. Lett. 101 (2012), p. 212110.

[5] C Ophus Microsc. Microanal. 25 (2019), p. 563.

[6] D Mukherjee et al., ACS Catal. 10 (2020), p. 5529.

[7] Y Han et al., Nano Lett. 18 (2018), p. 3746.

[8] X Hong et al., Microsc. Microanal. 27 (2021), p. 129.

[9] R Zhang et al., Microsc. Microanal. 26 (2020), p. 936.

[10] D Mukherjee and RR Unocic, Microsc. Microanal. 26 (2020), p. 2960.

[11] Research sponsored by the Hydrogen and Fuel Cell Technologies Office, Office of Energy Efficiency and Renewable Energy, U.S. Department of Energy (DOE). Electron microscopy was conducted at Oak Ridge National Laboratory's Center for Nanophase Materials Sciences (CNMS), a U.S. DOE Office of Science User Facility. 